NASA Technical Memorandum 88374

\title{
A Fast Data Acquisition System for the Study of Transient Events by High Repetition Rate Time-of- Flight Mass Spectromet ry
}

K.A. Lincoln and R.D. Bechtel

(NASA-TE-88374) A FAST CATA ACCOISITION SYSTEA FCB THE STODY CF TEALIENT EVENTS EY EIGH BEPETITICA EATE TIAE-OF-PIIGHT HASS

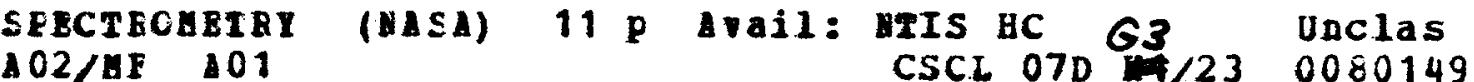

N87-24538

October 1986 


\section{A Fast Data Acquisition System for the Study of Transient Events by High Repetition Rate Time-of- Flight Mass Spectromet $r y$}

K. A. Lincoln, Ames Research Center, Moffett Field, California

R. D. Bechtel, Ferretec Inc., Milpitas, California 95035

October 1986

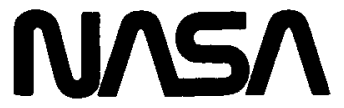

National Aeronautics and

Space Administration 
ERRATA

NASA Technical Memorandum 88374

A Fast Data Acquisition System for the Study of Transient

Events by High Repetition Rate Time-of-Flight Mass Spectrometry

K. A. Lincoln and R. D. Bechtel

Cover, title page, and COSATI page: Change the last word in the title from Spectrometer to Spectrometry.

Remove pages 7 and 8. Replace with new pages 7 and 8 , and add new page 9, which are provided. 
A EAST DATA ACQUISTION SYSTEM FOR THE STUDY OF TRANSIENT EVENTS BY HIGH REPETITION RATE TIME-OF-FLIGHT MASS SPECTROMET $Y$ Y

K. A. LINCOLN ${ }^{1}$ and R. D. BECHTEL ${ }^{2}$

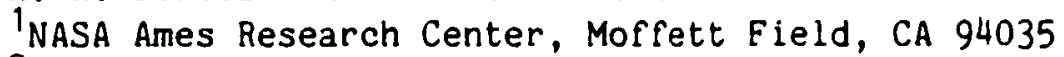

2Ferretec, Inc., 544 Gibraltar Dr., Milpitas, CA 95035

SUMMARY

Recent advances in commercially available data acquisition electronics embodying high speed $A / D$ conversion coupled to increased memory storage have now made practical (at least within time intervals of a third of a millisecond or more) the capturing of all of the data generated by a high repetition rate time-of-flight mass spectrometer producing complete spectra every 25-35 microseconds. Such a system has been assembled and interfaced with a personal computer for control and management of data. This paper describes its application to recording time-resolved spectra of individual vapor plumes induced from the pulsed-laser heating of material. Each laser pulse triggers the system to generate automatically a 3-dimensional (3-D) presentation of the time-resolved spectra with $\mathrm{m} / \mathrm{z}$ labeling of the major mass peaks, pius an intensity versus time display of both the laser pulse and the resulting vapor pulse. The software also permits storing of data and its presention in various additional forms.

\section{INTRODUCTION}

An earlier paper (ref. 1) pointed out the advantages of the time-of-flight mass spectrometer (TOFMS) associated with phenomena of rapidly changing gas compositions. It described several data acquisition techniques employing the state-of-the-art electronics at that time, and noted the limitations for capturing spectra owing to the then very limited amount of fast electronic memory storage. Subsequently, Holland, et al. (ref. 2) discussed at some length the requirememts for fast spectral recording in terms of applications on the chromatographic time scale. Now the availability of fast, compact waveform digitizers with megaword memories that can be readily coupled to a personal computer has made possible the capturing of all of the mass peaks on each cycle of the spectrometer (at least for short intervals of time), in a very manageable system. In addition, the computer makes possible a high degree of automation in the data-acquisition process including the tasks of mass calibration, logging pertinent experimental parameters, and presenting the recorded spectral data in several ways. The instrumentation described here is for the high ion current, high repetition rate TOFMS applications that produce analog signals requiring fast digitization for storage. 
OPERATIONAL FEATURES

The present computer-controlled data acquisition system has two options. The first accommodates the dynamic sampling of vapor plumes produced by the pulsed laser heating of various materials wherein time-resolved, multiple spectra are recorded in a waveform digitizer during all or part of the approximately $1 \mathrm{msec}$ vapor pulse resulting from each laser firing and the data are automatically transferred to the computer. Thus, after each firing of the laser, the equipment immediately displays 1) a 3-D (or "waterfall") video presentation of the time-resolved spectra from the vapor pulse, including $\mathrm{m} / \mathrm{z}$ labelling of the major mass peaks, 2) a second video presentation of both the laser intensity versus time and the total ion current pulse (from the TOFMS) versus time, and 3) a computer printout of all pertinent experimental parameters:

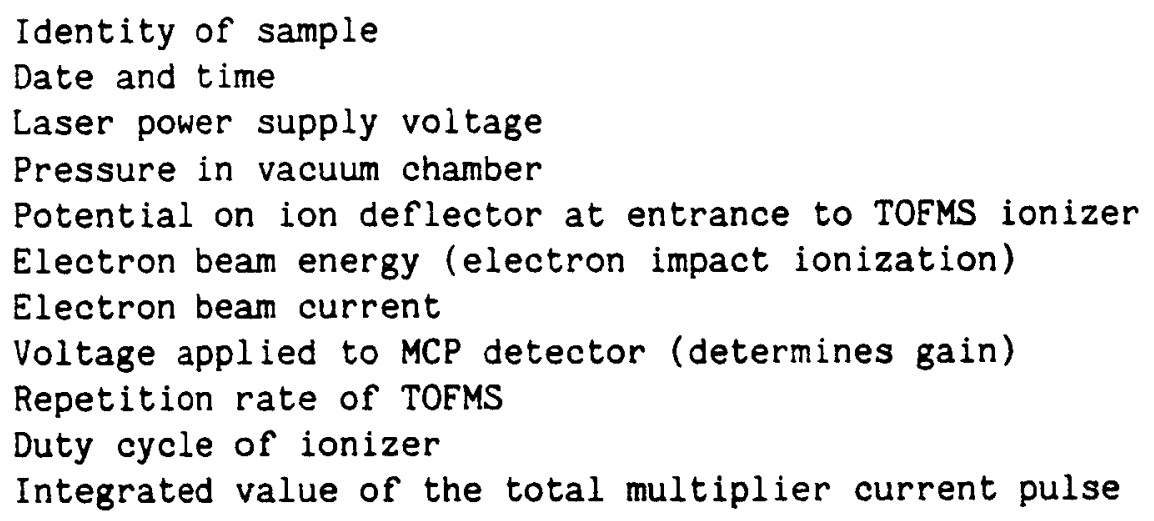

In addition to the above, the operator subsequently can call for hard copies of the following information from either the digital plotter or the computer printer:

The 3-D presentation of the spectra A spectrum plot resulting from averaging the 3-D spectra Plot of any expanded portion from either of above Plots of selected mass peaks vs. time derived from the 3-D spectra Tabulation of areas of each mass peak in the averaged spectrum normalized from the instrument parameters

The second option uses the same instrumentation to capture spectra over much longer periods of time, i.e., several tenths of seconds or more, to accommodate vapors from $\mathrm{CW}$ laser heating or calibration gases from a leak inlet. For these purposes the waveform digitizer is used in the fast signal-averaging mode to average in real time approximately 780 spectra/sec covering $\mathrm{m} / \mathrm{z} 1$ to 250 (or slower for higher mass ranges). Except for the 3-D feature on the first option, all of the above printouts and plots a available also in the second. 
EQU IPMENT

The laser/mass spectrometry system has been described earlier (ref. 3). It includes a CVC (Bendix) time-of-flight mass spectrometer with a nude EI source (but most of the electronics have been replaced with contemporary equipment). In our efforts to improve the data-acquisition capabilities and accomplish the above features, we have utilized computer automated measurement and control (CAMAC) digital electronics where feasible to save space and be able to readily link to a personal computer. The operation of the overall system can best be described by reference to Fig. 1 which is a simplified block diagram of the data-acquisition instrumentation. The TOFMS operates continuously at a preset repetition rate of 30 to $40 \mathrm{kHz}$ and sends out a trigger pulse coincident with the onset of each cycle. The output of the microchannel plate detector (ref. 4) in the TOFMS is split into the ac component containing the spectral signals and the dc component or total multiplier current (TMC) output which is a convenient measure of all of the masses in the vapor.

For pulsed laser operation the TMC is amplified and, along with the laser pulse, is fed to a DATA 6000 Waveform Analyzer (Data Precision, Danvers, MA). Thus, both the laser pulse and the vapor pulse are displayed on the DATA 6000 screen. Simultaneously, the spectra are amplified and continuously fed to a scope for visual monitoring and also to a transient recorder which is set in the single-shot mode but is not triggered (because of the gate) until the arrival of the first TOFMS trigger pulse following a preselected time delay after the laser pulse. This optimizes the recording of multiple spectra to coincide with the arrival of the vapor pulse at the TOFMS. At that time, the transient recorder begins to store consecutive spectra until its memory is full, and the stored data is synchronized with the beginning of a spectrum (the latter is necessary for subsequent $\mathrm{m} / \mathrm{z}$ calibration of the spectra). For longer sampling periods (such as with the $\mathrm{CW}$ laser), the transient recorder does the digitizing but it is operated in the averaging mode in concert with the signalaveraging memory module which is set for a preselected number of spectra (up to $65,536)$ to be averaged. The transient recorder is then triggered by the operator (or computer) to produce the averaged spectrum.

The model $2001 \mathrm{~S}$ Transient Recorder, the model 4100 Signal Averaging Memory, the model 2032 Scanning Digital Voltmeter (DVM), and the model 6001 Crate Controller (containing the microprocessor) are all CAMAC modules (DSP Technology, Inc.--formerly Transiac--Fremont, CA) housed in a CAMAC crate and communicate via the CAMAC dataway to an IBM-XT computer. The memory content of the transient recorder (or signal-averaging memory) is automatically dumped into the computer which is programed to display on its monitor the spectra in the 3-D format for pulsed work or 2-D averaged spectra for continuous mode applications. The $2001 \mathrm{~S}$ is an 8-bit digitizer with a 100-MHz maximum sampling 


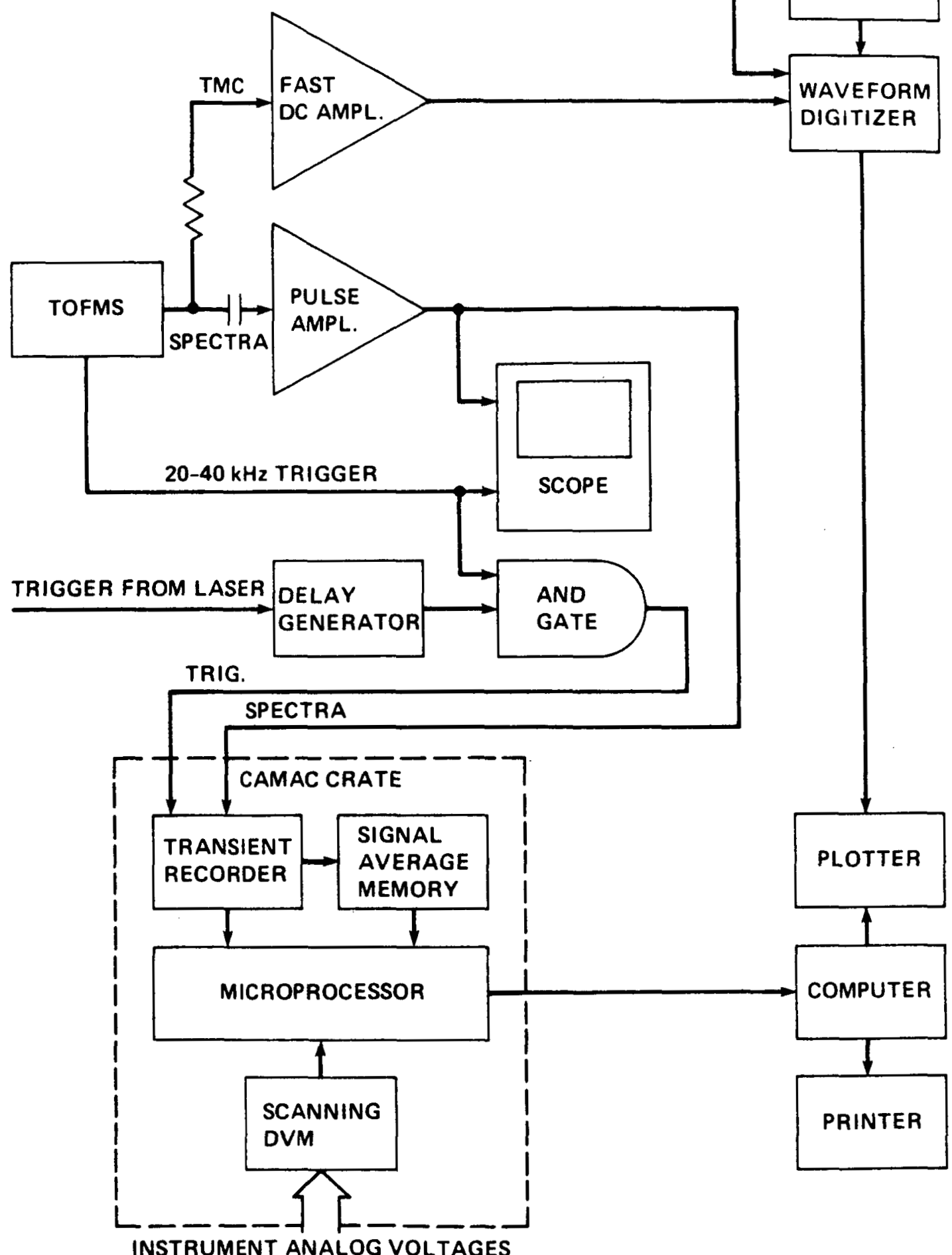

Fig. 1. Data Acquisition Instrumentation

rate and a 32-kilobyte memory; thus, there is a tradeoff between the best resolution ( 10 nanosec/sample), the mass range, and the number of spectra that can be recorded. In some applications, switching to 20 nanosec/sample and 
doubling the number of spectra stored is preferred. Also, it is possible to make more efficient use of the limited memory in the transient recorder by using a burst clock generator in place of the internal sampling clock; in this way only those portions of the spectrum that are of interest are stored in the memory (ref. 1). This latter technique is not needed in the signal-averaging mode, because the signal averaging memory is $8 \mathrm{k}$ which will accommodate a very large mass range.

SOFTWARE AND DESIGN FEATURES

The main programing language used in the software design is MS-Fortran (which interfaces with MS-Pascal), Multihalo (Media Cybernetics Inc.), CAMV3 (Transiac Dataway Communication Driver), and GPIB subroutines. Because of the limitations of Fortran, the Pascal function DOSXQQ is utilized to recognize IBM-XT function-key operations, thus allowing for simple menus. The 3-D, 2-D, and averaged presentation of the data are achieved by using the Multihalo drivers which essentially allows the program to draw lines from point to point. Expansion and contraction of the waveform data utilizes a cursor feature of the Multihalo that allows the Fortran program to read the new spectra form simply and efficiently via function-key operations. The program consists mainly of five blocks allowing for instrument set-up, data acquisition or transfer, video display, plotting, and calibration.

First, all of the instruments are programmed for data acquisition; then after the laser is fired and the spectral data are recorded (or when in continuous mode after the averaging is completed), the program automatically transfers the data from the CAMAC instruments to the computer. In the 3-D mode the program then averages the several spectra (usually 10 or more) so that no mass peaks will be overlooked in the mass labelling routine. The routine achieves mass labelling of the major peaks by searching the averaged spectrum for peaks that exceed a preset threshold percentage of the maximum mass peak intensity and effecting a time-to-mass conversion for them via the relationship

$t_{f}=k_{1} \sqrt{m / 2}+k_{2}$

where $k_{2}$ accounts for any delays in the system. The program then generates the desired video displays, 3-D, 2-D, etc. per prior menu choice in the setup section. The constants, $k_{1}$ and $k_{2}$, used in the time-to-mass conversion are calculated in the calibration section of the program. They are acquired by leaking a calibration gas into the spectrometer and displaying its 2-D spectrum on the video terminal; the mass peaks are identified by the operator and their $\mathrm{m} / \mathrm{z}$ values entered into the program. After several peaks are so identified, the 
program calculates the constants using a least squares iteration and stores them in a file for subsequent use.

\section{RESULTS AND DISCUSSION}

Figures 2 through 5 are examples of some of the graphic outputs of the system. All of them were obtained from the same laser pulse focused on a graphite sample positioned $12 \mathrm{~cm}$ from the electron beam in the TOFMS. Figure 2 is an intensity-time presentation of the laser pulse and the resulting vapor pulse;
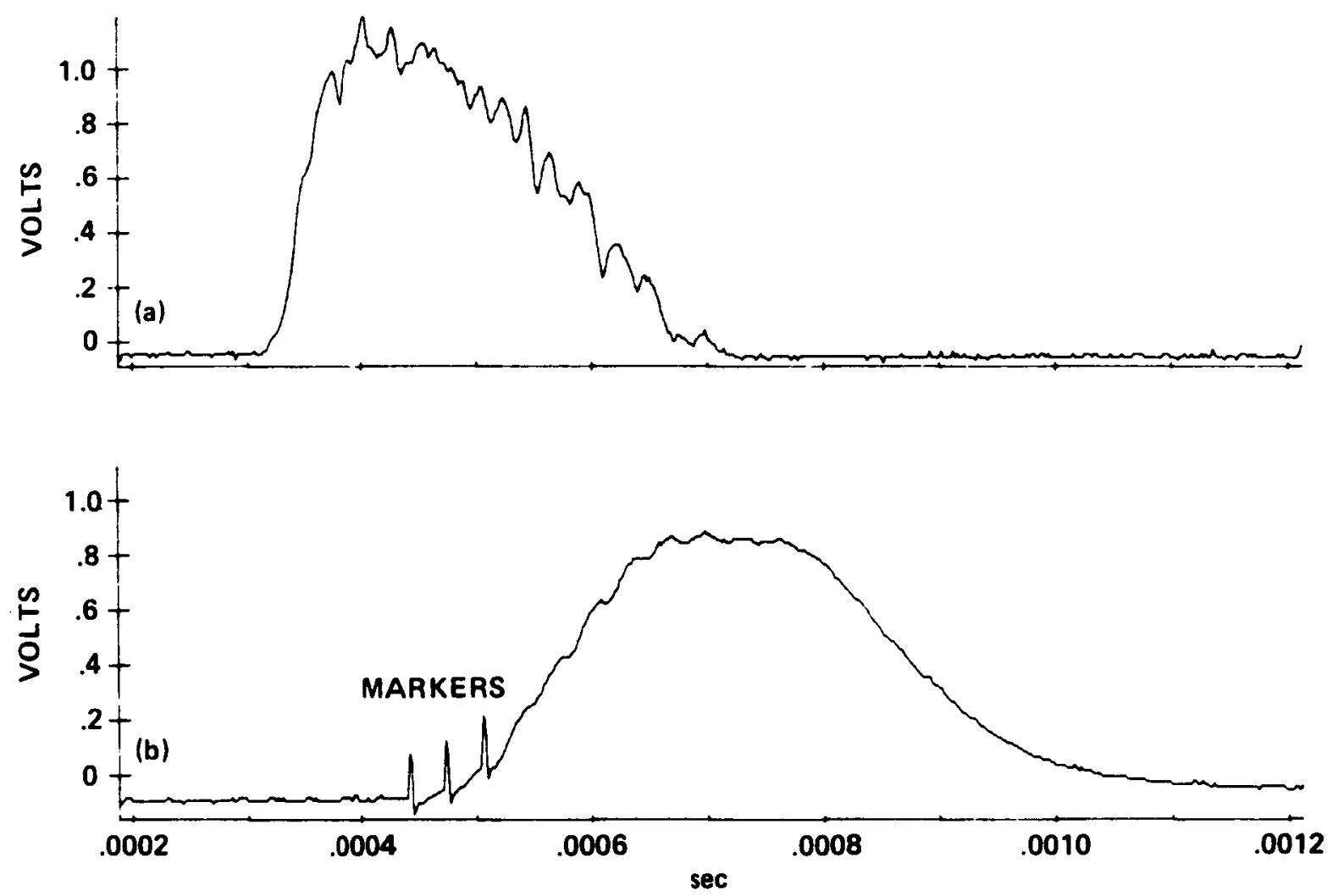

Fig. 2. Intensity vs. time of (a) laser pulse and (b) vapor pulse.

it is a plot of the video display from the DATA 6000 . Figure $2 b$ is the microchannel plate output current amplified by a fast differential DC amplifier (Hewlett-Packard 8875A) that has an adjustable filter which smooths out the individual pulses that comprise the total ion current in the spectra. The markers in Fig. $2 b$ indicate where during the vapor pulse the transient digitizer began recording the spectra shown in Fig. 3b. Figure $3 a$ is the printout of the instrument parameters from the laser shot, and Eig. $3 b$ is a printout of the 3-D presentation that automatically appears on the computer video display. Any portion of it can be selected for expansion and plotted as shown in Fig. 4. The time-resolved feature shows the carbon species at masses 12,24 , and 36 going through a maximum while the stable gases, $\mathrm{C}_{2} \mathrm{H}_{2}(26)$ and $\mathrm{CO}(28)$, are continuing to increase during this recorde' interval. Figure 5 is the 
LASER MASS SPECTROMETER PARAMETERS

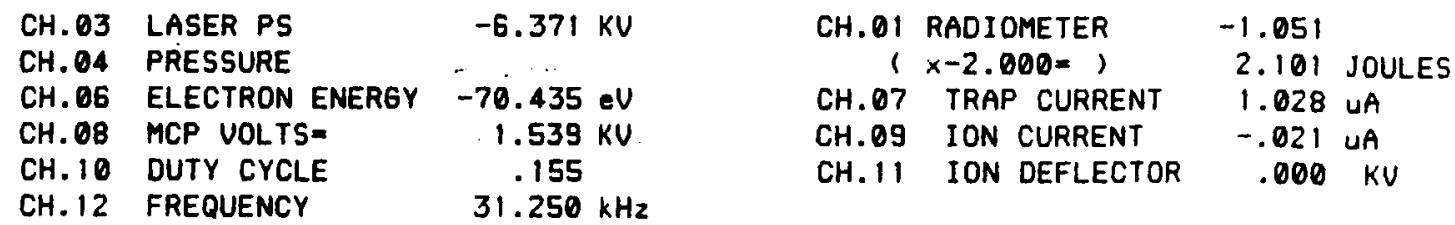

(a)

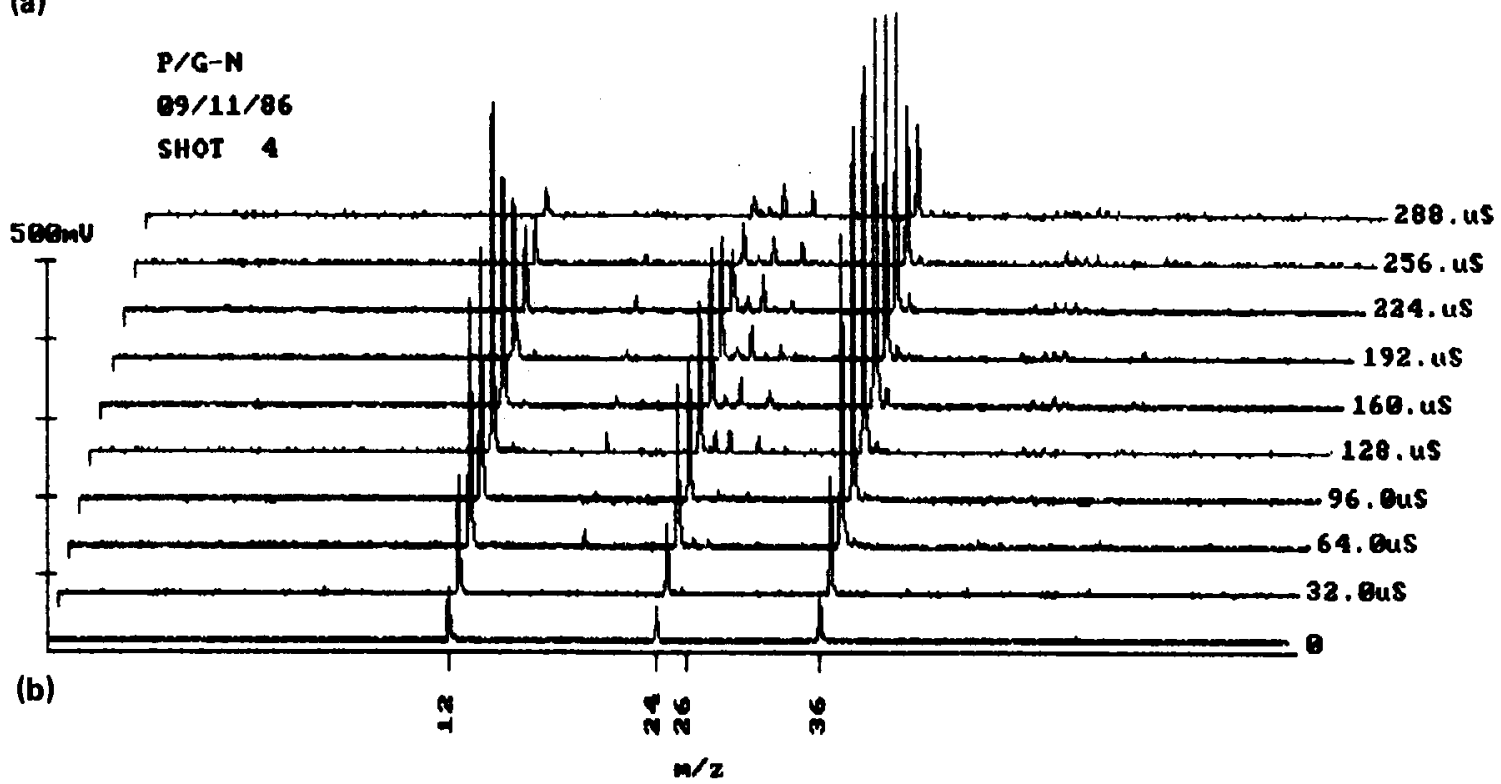

Eig. 3. Immediate computer outputs. (a) Printout of instrument parameters. (b) Screen printout of 3-D video display.

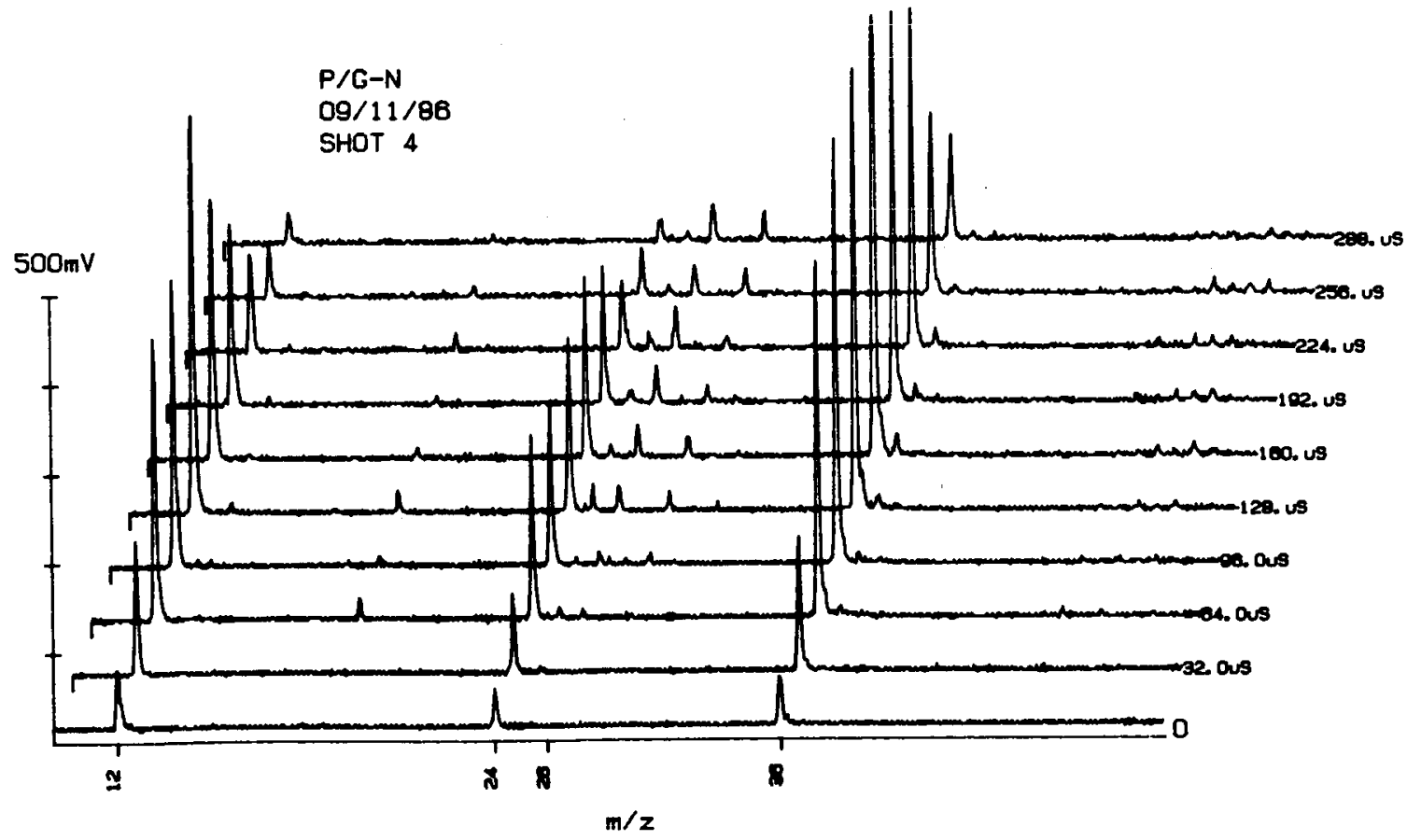

Fig. 4. Plot of 3-D spectra within a selected mass range. 


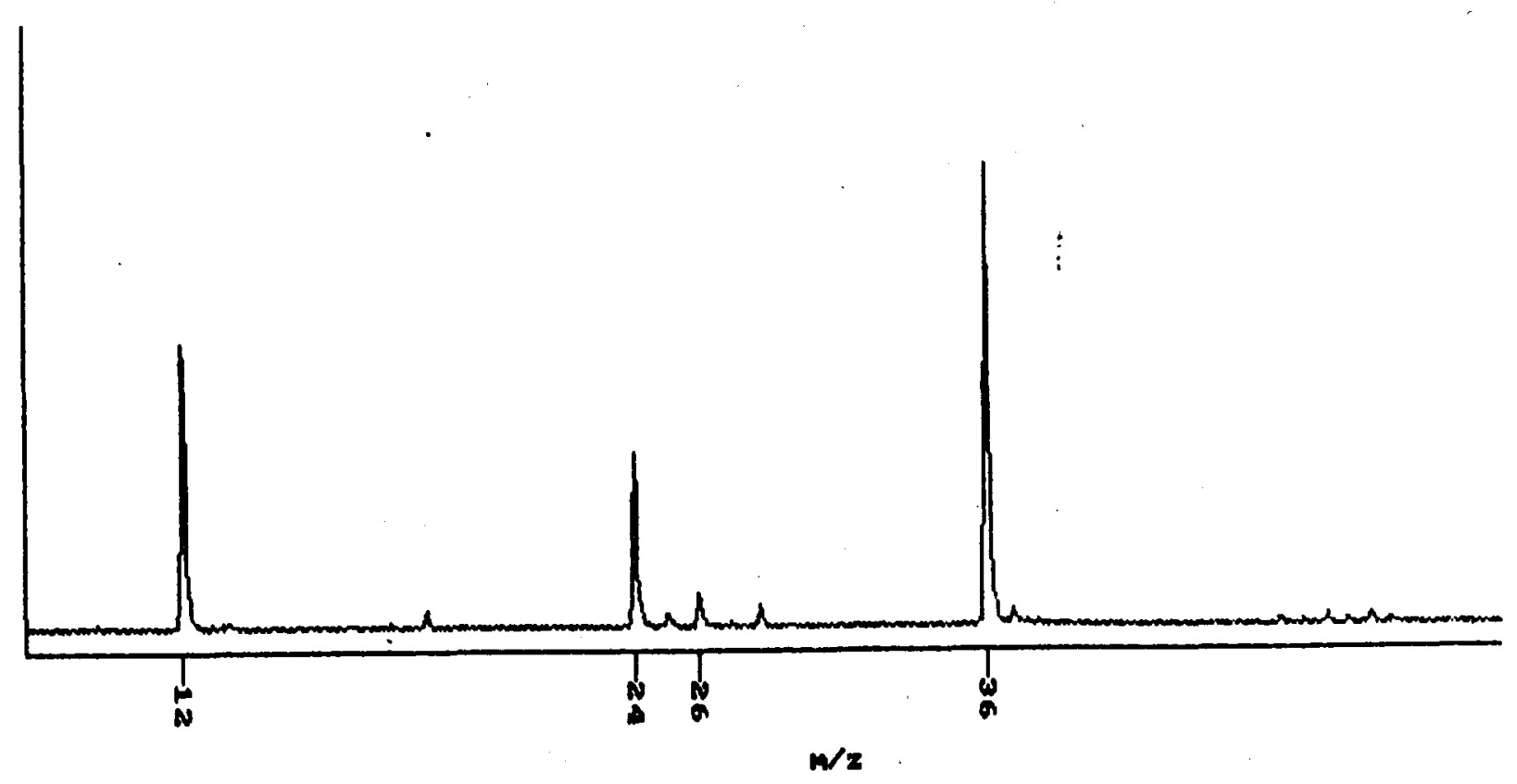

Fig. 5. Screen printout of the average of the time-resolved spectra.

averaged spectrum from the ten spectra of Fig. 4. The array from which it is obtained is used in the program as a basis for the mass labelling; furthermore, the area under each of these mass peak is available to provide a more quantitative measurement of the vapor species.

Inasmuch as the 3-D spectra are already stored in the computer and displayed, the program permits the operator selection of any $m / z$ values of interest and plots them vs. time. An example of this is Fig. 6 which is a timeresolved display of three $\mathrm{m} / \mathrm{z}$ values resulting from a single laser shot. These are not plots of peak heights vs. time, but rather, the areas under the individual peaks vs. time, thus providing a more accurate presentation of the variations in vapor composition.

Current progress in electronics that has now made CAMAC modules available which feature $200 \mathrm{MHz}$ sampling rates and megaword memories is setting a trend for the future which indicates that the present equipment is not a limitation for the data acquisition technique described here. 


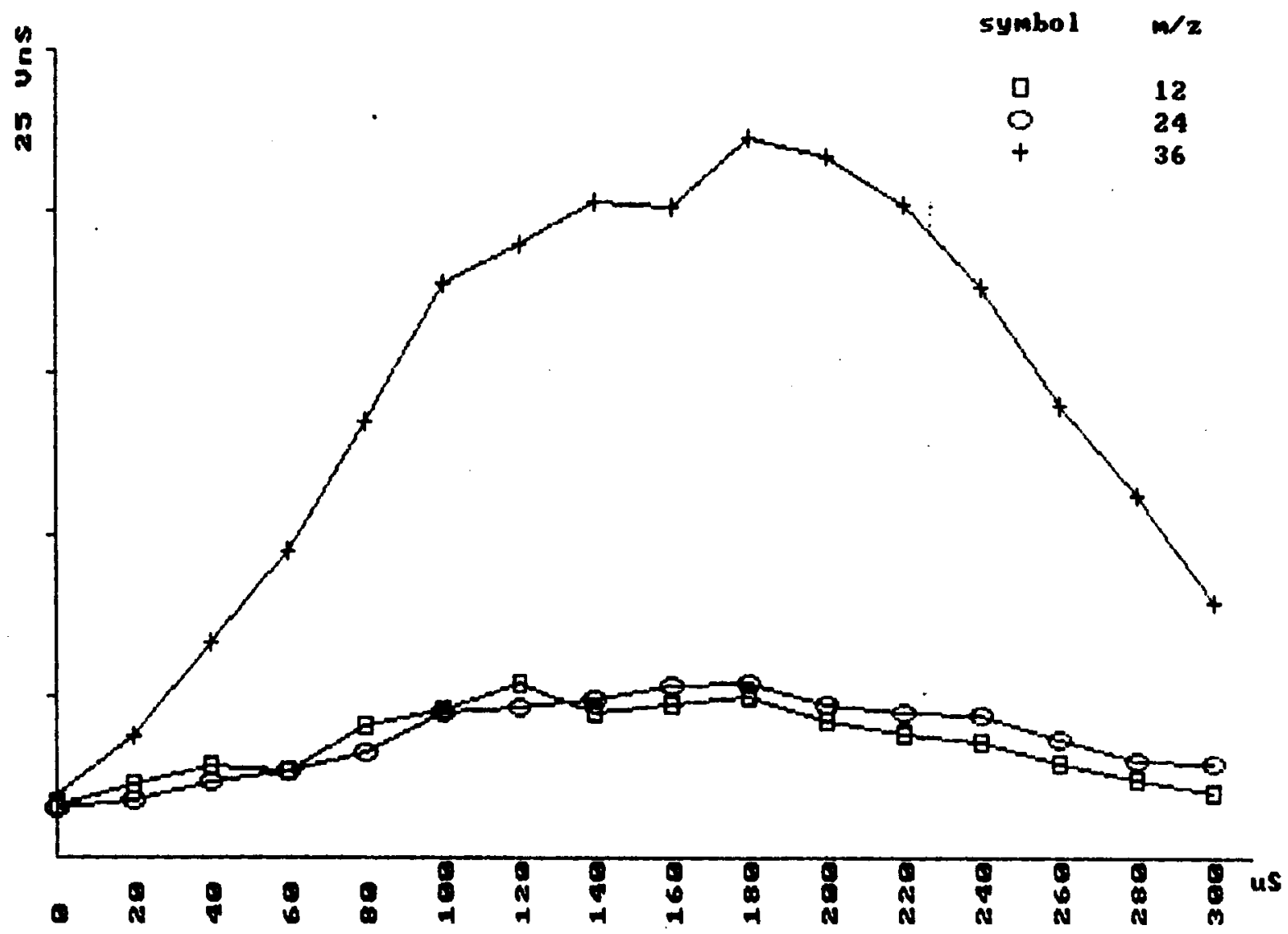

Fig. 6. Screen printout of three post-selected $\mathrm{m} / \mathrm{z}$ values vs. time.

\section{ACKNOWLEDGMENT}

Appreciation is extended to Miguel Mateos who assisted with the computer programming and the acquiring of data.

\section{REFERENCES}

1 K.A. Lincoln, Data Acquisition Techniques for Exploiting the Uniqueness of the Time-of-Flight Mass Spectrometer: Application to Sampling Pulsed Gas Systems, in: D. Price and J.F.J. Todd (Eds.), Dynamic Mass Spectrometry, Vol. 6, Heyden \& Son, London, 1981, pp. 111-119.

2 J.F. Holland, C.G. Enke, J. Allison, J.T. Stults, J.D. Pinkston, B. Newcome and J.T. Watson, Mass Spectrometry on the Chromatographic Time Scale: Realistic Expectations, Anal. Chem., 55(9) (1983) 997A-1012A.

3 K.A. Lincoln, Techniques for Avoiding Discrimination Errors in the Dynamic Sampling of Condensable Vapors, Int'1. J. Mass Spectrom. and Ion Pron.., 60 (1984) 165-172.

4 K.A. Lincoln, Paper presented at the 32nd Annual Conference on Mass Spectrometry and Allied Topics, San Antonio, Texas, May 27-June 1, 1984. 


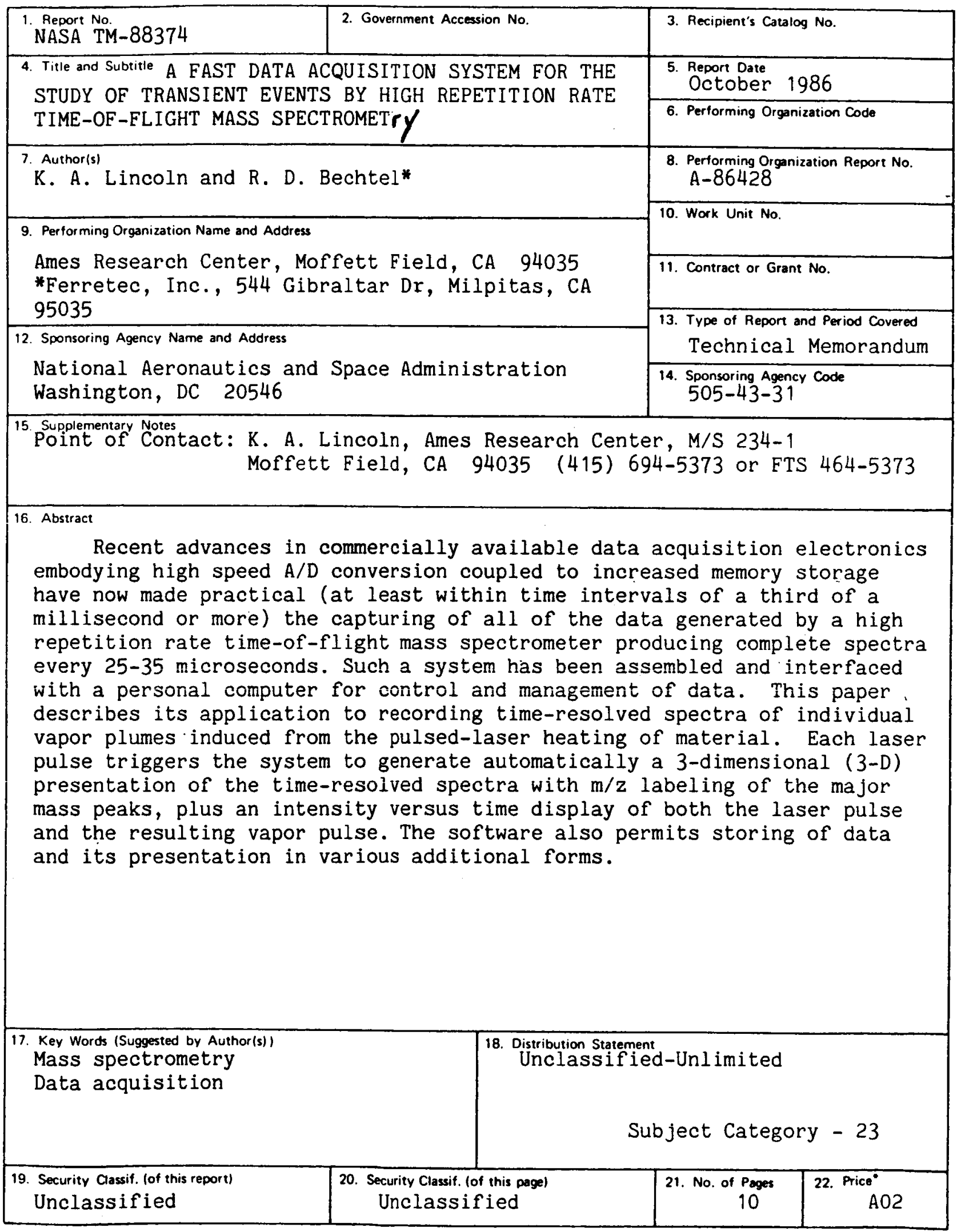

"For sale by the National Technical Information Service, Springfield, Virginia 22161 